\title{
Genetics in Dentistry
}

\author{
Sugandha Dadhich and Gargi Jani* \\ Dental Practitioner, Ahmedabad, Gujarat, India \\ *Corresponding Author: Gargi Jani, Dental Practitioner, Ahmedabad, Gujarat, India.
}

Received: March 02, 2020

Published: May 08, 2020

(C) All rights are reserved by Sugandha

Dadhich and Gargi Jani.

\begin{abstract}
The article reviews the literature on the genetic aspects of various disorders affecting the oral cavity and highlights the influence of genetics on diseases encountered in routine practice. Understanding the mechanism of a disease at genetic level will help an oral health professional in early intervention and would also help to explain a patient why persons with same environmental and behavioral (oral habits, diet) risks are relatively more predisposed to a certain disease.
\end{abstract}

Keywords: Genetics; Dentistry; Mutation; Gene

\section{Introduction}

Genetics is the study gene, genetic variations, hereditary of living organism. It is the field of biology intersecting with various life sciences and strongly associated with the study of information system $[1,2]$.

The study is done at all the levels from molecules to population. The basic study of genetics includes:

- $\quad$ Study of gene, DNA, chromosome.

- Inheritance and its various patterns.

- Genetic mutation.

- Genetic variation.

- Genes and environmental interplay.

- $\quad$ Family medical history and genes.

Gene is the basic unit of hereditary; it is the segment of DNA that is passed down from parents to off springs. It is responsible for protein formation, cell structure and function. DNA is a double stranded structure that contains complementary genetic information. During replication of DNA (s phase) if there is matching error in the complementary structure, a protein repairs it and keeps the genome intact but with faulty repair protein errors accumulate leading to genetic mutation: a permanent alteration. Genetic mutation is of two types:
- Hereditary: Inherited from a parent and present throughout a person's life and present in every cell of the body.

- Acquired: Occur at some point during person's life due to environmental factors and present in certain cells.

During mutation of DNA, in DNA codon frameshift mutation occurs due to substitution, insertion or deletion resulting in defective protein which are either inactive or disease causing.

Tooth is a specialized unit of maxillofacial skeleton formed by a series of complex steps. Tooth development is under genetic control and is regulated by inductive interactions between epithelial and mesenchymal cells $[1,2]$. The contribution of hereditary factors in the pathogenesis of dental caries, periodontal problems, dental anomalies and other oral and maxillofacial disorders are becoming increasingly evident in dentistry. Identification of underlying cause of a condition starts with localization of its defective gene in human genome. Progression made by human genome project over the years has greatly increased the feasibility of mapping inherited condition.

The role of genetics has been increasingly recognized in the understanding of various dental diseases and anomalies.

The aim of this article is to highlight the influence of genetics on various dental disorders that are encountered in day-to-day practice. 
Genetics and dental anomaly

Role of genetics in tooth agenesis

Tooth Agenesis is the most common developmental dental anomaly. Agenesis is characterized by congenital absence of one or more teeth. The incidence for permanent tooth agenesis ranges from $1.6 \%$ to $9.6 \%$ in the general population excluding third molars [3-7].

Various terminologies are used in literature to describe this numeric anomaly. Hypodontia is absence teeth but not more than six (excluding $3^{\text {rd }}$ molars). Oligodontia is used to describe absence of six or more teeth (excluding $3^{\text {rd }}$ molars) in dental arches. Anodontia is an extreme expression of oligodontia denoting complete absence of teeth.

Population studies have shown that tooth agenesis can be manifested as an isolated finding or part of a syndrome [8,9]. Grahnen has proposed that tooth agenesis is typically transmitted as an autosomal dominant trait with incomplete penetrance and variable expressivity [5]. Several genes have been investigated as a candidate for isolated tooth agenesis but mutations occurring in MSX1 and PAX9 are shown to be involved in non-syndromic tooth agenesis. A familial autosomal dominant hypodontia was noted by a point mutation in the MSX1 gene. Vastardis., et al. [10] using a linkage analysis in a family with second premolar and third molar agenesis, demonstrated a locus on the chromosome 4p16 as the site of the MSX1. Arg31-to-pro mutation influences MSX1interactions, which are critical for normal tooth development. On sequence analysis arg31-to-pro missense mutation was demonstrated in the MSX1 homeodomain for all of the affected subjects [10].

Another gene causing tooth agenesis is Pax9 in chromosome 14 (14q21-q13). The frameshift mutation of Pax9 was identified for creating autosomal dominant pattern of oligodontia in a family for four generations. Nieminen., et al. identified an A-to-T transversion of the Pax9 gene in a family with autosomal dominant oligodontia [11]. Mutation of AXIN2 gene is responsible for creating autosomal dominant oligodontia which is also known for creating colon cancer.

Role of genetics in structural anomalies

The structural anomalies of teeth are caused due to disturbances created during the development of enamel and dentin.

Amelogenesis imperfect is a disorder caused by disturbance during enamel formation. Amelogenesis imperfecta is of three types:
1. Hypoplastic: Thin, normally calcified enamel,

2. Hypocalcified: Less mineralized, but normal thickness enamel

3. Hypomaturation: Enamel structure having the same radiodensity of dentin and can be easily separated from dentin.

Amelogenesis imperfect exhibits X-linked, autosomal dominant and recessive inheritance caused by mutation of:

- AMELX gene: Associated with enamel protein amelogenin. Mutation of AMELX is correlated with hypoplastic and hypomaturation variants.

- $\quad$ ENAM gene: Associated with enamel protein enamelin. Mutation of ENAM is correlated with autosomal dominant and recessive patterns of hypoplastic variants.

- $\quad$ MMP20: Codes for enamelysin, a proteinase; mutation of this gene is associated with autosomal recessive pattern causing pigmented hypo maturation variant.

- KLK4: Is associated with protease kallilerin-4, the mutation of which results in various forms of hypo maturation amelogenesis imperfecta.

- DLX3: Codes for various proteins that are critical for craniofacial, tooth, hair, brain and neural development; mutation leading to hypoplastic-hypo maturation variants.

Dentinogenetic imperfecta; also known as Capdepont's teeth dentin is a hereditary developmental disturbance of dentin in absence of any systemic disorder. It is an autosomal dominant condition with three variations: Type I is syndromic form of DGI which is inherited with osteogenesis imperfecta and the genes encoding collagen, type I, alpha 1, COL1A1 and COL1A2 [13]. The other two forms are the result from mutations in the gene dentin sialo phosphoprotein (DSPP), encoding dentin phosphoprotein and dentin sialoprotein $[12,13]$. Dentinogenesis imperfecta formerly divided into hereditary opalescent dentin and Brandywine isolate, where the later representing unusual pulpal enlargement also known as shell teeth. Current evidences suggest that Brandywine isolation is the variable expressivity of the gene for dentinogenesis imperfecta. Investigators have documented enlarged pulp chambers in the affected individuals whose parents and children represent classic DGI.

\section{Genetics and periodontal disease}

Periodontal disease is multifactorial in which both genetic and environmental factors play an important role, whereas microbiota is responsible for the progression of disease. A range of host genetic factors can influence individual susceptibility to periodontal 
disease and are able to influence the clinical aspects and rate of progression of the disease [14]. The new evidences has shown that $\mathrm{PD}$, like other diseases (diabetes, cancer, etc.), requires a genetic predisposition [14].

Individuals respond differently to oral microflora according to their genetic predisposition. Also, genetics determines the interaction of a person with environmental factors in the onset of periodontal disease. It is thought that about $50 \%$ of the probability of developing PD is related to heredity [15]. Genetic susceptibility to multifactorial diseases is usually due to several gene polymorphisms. Common variation in the genetic code may results in altered expression or in functional changes of the encoded proteins, therefore resulting in an increase of disease severity or making individuals with genotypes more susceptible to a given disease $[14,16]$. Recent investigations on susceptibility factors of periodontitis have mainly focused on genes that modulate immune response such as cytokines, chemokines, cell surface receptors, enzymes and proteins related to antigen recognition [14]. Cytokines, such as IL- $1 \alpha$, IL-1 $\beta$, IL-10 and IL-6, are key factors which mediate the inflammatory process during the progression of periodontitis. They have a role in activation, proliferation and differentiation of $B$ cells that are the infiltrating cells in advanced periodontitis lesions $[14,17]$. Thus, common variations in genetic code can alter the progression of disease $[14,18]$.

Role of genetics in caries susceptibility

Dental caries is a chronic, complex, multifactorial disease affected by local (saliva, microbiota), environmental (fluoridation) and genetic factors. The disease is prevalent in all age groups.

Increased enamel porosity, decreased mineral content, and enamel crystal inhibitory proteins are well known that lead to increased caries development. Genes that regulate the immune complex have been associated with increased colonization of cariogenic bacteria and enamel defects, which shows probability of linking specific allelic variation and caries susceptibility in the general population [19]. Hence the various gene associated with immunity and during the development of tooth are responsible for caries.

\section{Role of genetics in oral cancer}

Oral cancer is one of the leading causes of death in developing countries; the disease being more prevalent in heavy smokers, tobacco and alcohol addicts. It is likely that more than one factor is needed to produce cancer. Extrinsic factors include tobacco smoke, alcohol, syphilis, sunlight, radiation etc. Intrinsic factors general malnutrition or iron deficiency anemia, gene mutation.
Oncogenes and tumor suppressing genes are chromosomal components capable of being affected by variety of causative agents. Normal genes or proto-oncogenes are transformed into activated oncogenes through three mechanisms (I) Point mutations in a proto-oncogenes that result in a constitutively acting protein product, (ii) localized reduplication (gene amplification) of a DNA segment that includes a proto-oncogenes leading to overexpression of the encoded protein, (iii) chromosomal translocation that brings a growth-regulatory gene under the control of a different promoter and that causes inappropriate expression of the gene [26].

Once activated, they may stimulate the production of excessive amount of new genetic material through amplification of involved gene. It has been supposed that oncogenes are involved in initiation and progression of wide range of neoplasms.

Whereas tumor suppressor genes allow the tumor production indirectly when they become inactive or mutated.

Commonly identified genetic deviance in oral squamous cell carcinoma include abnormality of ras, myc and EGFR oncogenes and p53, pRb, p16 and E-cadherin tumor suppressor genes. According to researcher's accumulation of several genetic aberrations is necessary before the affected cell expresses malignant phenotype.

Carcinogenesis is a complex, multi-process in which genetic events within signal transduction pathways governing normal cellular physiology are quantitatively altered [20]. Cancer is the result of accumulation of changes in the excitatory and inhibitory cellular pathways, which may occur at any level of a given pathway. It has been estimated that from three to six somatic mutations are needed transform a normal cell into its malignant counterpart [20]. For human oral cancer more than 63 karyotypes have been described. Among them loss of chromosome 9, 13, 18 and Y deletions are more commonly reported than others [21]. Approximately two-third of all head and neck cancer cells contain a deleted region located in chromosome 9p21-22, which appears in dysplastic and carcinomain-situ lesions [22-24].

Smoking and tobacco use have been associated with the mutation of the p53 gene in squamous cell carcinoma of the head and neck. By immunohistochemistry p53 expression has been shown in oral tumors from patients who were heavy smokers and drinkers [25].

\section{Dental genetic test}

With available knowledge and techniques, dental genetic tests are possible by taking saliva or cheek swab. In certain test blood samples are also taken. The purpose of genetic test is to: 
- Identify increased risks of health problems.

- $\quad$ Choose treatments.

- Assess responses to treatments.

The available types of genetic test are:

- Diagnostic: To identify the disease gene.

- Predictive: To identify gene that increases the chance of developing disease.

- Carrier testing: To find a person having a gene responsible for a disease.

- Pharmacogenomic: To get information how certain medicines are processed by individual's body.

- Prenatal: Done during pregnancy to find if the fetus has certain disease.

- Newborn screening.

From dental genetic test one can confirm the disease, identify genes that are responsible for a disease and genes that could be passed on to offspring, determine severity of disease, guide in deciding the course of treatment and best medicine for certain individual and screen newborns for avoidable and treatable conditions.

\section{Conclusion}

Dentists are the first line of defense to oral pathologies and implication of complex diseases of oral cavity and head and neck regions. It has been established through various studies that gene mutations are known to play a key role in various oral health problems. Therefore it is important for an oral health professional to understand the advances in molecular biology and genetics for early detection of a high risk individual, create awareness and educate the individual, thus incorporating early intervention strategy, enforcing strict oral habits, proper diet and thereby enhancing the quality of life. With the knowledge genetics, a health professional is capable of providing a more personalized level of care. With advancing technology and increasing knowledge the future of personalized medicines is near where the patient would be given medicines according to his genetic constitution. Also, this field offers great scope of further research.

\section{Bibliography}

1. Thesleff I., et al. "Regulation of organogenesis. Common molecular mechanisms regulating the development of teeth and other organs". The International Journal of Developmental Biology 39 (1995): 35-50.

2. Thesleff I and Niemen P. "Tooth morphogenesis and cell differentiation". Current Opinion in Cell Biology 8 (1996): 844-850.
3. PindborgJJ. "Abnormalities of tooth morphology". In: Pathology of the dental hard tissues. Copenhagen: Munksgard (1970): 15-74.

4. Clayton JM. "Congenital dental anomalies occurring in 3,557 children". Journal of Dentistry for Children - AAPD 23 (1956): 206-208.

5. Grahnen H. "Hypodontia in the permanent dentition: a clinical and genetical investigation". Odont Revy 7 (1956): 1-100.

6. Eidelman E., et al. "Hypodontia: prevalence amongst Jewish populations of different origin". American Journal of Physical Anthropology 39 (1973): 129-133.

7. Bergstrom K. "An orthopantomographic study of hypodontia, supernumeraries, and other anomalies in school children between the ages of 8-9 years: an epidemiological study". Swedish Dental Journal 1 (1977): 145-157.

8. Jorgenson RJ. "Clinician's view of hypodontia”. Journal of the American Dental Association 101 (1980): 283-286.

9. Gorlin RJ., et al. "Syndromes of the Head and Neck". New York: Oxford University Press (1990).

10. Vastardis H., et al. "A human MSX1 homeodomain missense mutation causes selective tooth agenesis". Nature Genetics 13 (1996): 417-421.

11. Nieminen P., et al. "Identification of a nonsense mutation in the PAX9 gene in molar oligodontia". European Journal of Human Genetics 9 (2001): 743-746.

12. Xiao S., et al. "Dentinogenesis imperfecta 1 with or without progressive hearing loss is associated with distinct mutations in DSPP". Nature Genetics 27 (2001): 201-204.

13. Cakan DG., et al. "The genetic basis of dental anomalies and its relation to orthodontics". European Journal of Dentistry 7.1 (2013): S143-S147.

14. Carinci F., et al. "Genetic Risk Assessment of Periodontal Disease during Dental Treatments". Anneals Forensic Research Analyst 2.1 (2015): 1011.

15. Michalowicz BS., et al. "Evidence of a substantial genetic basis for risk of adult periodontitis". Journal of Periodontology 71 (2000): 1699-1707.

16. Vijayalakshmi R., et al. "Genetic polymorphisms in periodontal diseases: an overview". Indian Journal of Dental Research 21 (2010): 568-574. 
17. Michalowicz BS., et al. "Evidence of a substantial genetic basis for risk of adult periodontitis". Journal of Periodontology 71 (2000): 1699-1707.

18. Loos BG., et al. "Identification of genetic risk factors for periodontitis and possible mechanisms of action". Journal of Clinical Periodontology 32.6 (2005): 159-179.

19. Bretz WA., et al. "Evidence of a contribution of genetic factors to dental caries risk". The Journal of Evidence-Based Dental Practice 3.4 (2003): 185-189.

20. Vogelstein B and Kinzler KW. "The multistep nature of cancer". Trends in Genetics 9 (1993): 138-141.

21. Jurel SK., et al. "Genes and oral cancer". Indian Journal of Human Genetics 20.1 (2014): 4-9.

22. Ah-See KW., et al. "An allelotype of squamous carcinoma of the head and neck using microsatellite markers". Cancer Research 54 (1994): 1617-1621.

23. Nawroz H., et al. "Allelotype of head and neck squamous cell carcinoma". Cancer Research 54 (1994): 1152-1155.

24. Van der Riet P., et al. "Frequent loss of chromosome 9p21-22 early in head and neck cancer progression". Cancer Research 54 (1994): 1156-1158.

25. Langdon JD and Partridge M. "Expression of the tumour suppressor gene p53 in oral cancer". British Journal of Oral and Maxillofacial Surgery30 (1992): 214-220.

26. Krishna A., et al. "Molecular concept in human oral cancer". National Journal of Maxillofacial Surgery 6.1 (2015): 9-15.

\section{Assets from publication with us}

- Prompt Acknowledgement after receiving the article

- Thorough Double blinded peer review

- Rapid Publication

- Issue of Publication Certificate

- High visibility of your Published work

Website: www.actascientific.com/

Submit Article: www.actascientific.com/submission.php

Email us: editor@actascientific.com

Contact us: +919182824667 